\title{
We Hear With our Brain as the same as our Ears
}

\author{
Alireza Bina* \\ Starwood Audiology, USA \\ *Corresponding author: Alireza Bina, Starwood Audiology, USA, Email: bina_alireza@yahoo.com
}

\begin{abstract}
There are some studies which confirmed that dysfunction in Central Nervous System (CNS) may cause a malfunction in the Peripheral Auditory system (Cochlea_Auditory Nerve, Auditory Neuropathy), but the question is could Brain Disorder without any lesion in Cochlea and/or Auditory nerve cause Sensorineural Hearing Loss? It seems that there are a lot of Sensorineural hearing loss which they have neither Sensory nor Neural lesion, Brain is involved causing them. We deal with this subject in this paper and we propose a new theory that External Ear Canal is not the only input of Auditory Signals, Sounds could receive by the head and Cerebral Cortex and approach to the Cochlea (Backward Auditory input of Sounds).
\end{abstract}

\section{Discussion}

There are some studies that verified Otosclerosis and Meniere Diseases which are Peripheral pathologies initiated from CNS [1,2]. Increasing Cortisol Level and lack of Dopamine in Hypothalamus is involved in Meniere Disease and in Otosclerosis infection of CNS by Measles Virus [1,2]. According to these studies medications such as Ribavirin, Interferon, Inosine Pranobex with or without other medications such as, bisphosphonates, Sodium Fluoride with Calcium may be a cure or treatment of Otosclerosis and Dopamine Agonist Medication may be helpful for the treatment of Meniere Disease, further studies need to be done to find out the usefulness of these medications for these disorders and side effect, interaction and so on $[3,4]$. There are some papers which indicated Auditory Neuropathy occurred following Inferior Colliculus Disorder and Sudden Deafness following Primary Auditory Cortex infarction so it seems Central Auditory processing disorders may cause damage in Peripheral Auditory system, however, there are some patients with normal Audiogram(normal peripheral hearing) but they suffer from Central Auditory Processing Disorder(CAPD) such as Autism spectrum, therefore, malfunction in CNS may cause peripheral hearing loss but it depends on the site and degree of Lesion in the CNS, probably mild lesion in some part of CNS does not affect peripheral Auditory system [5-13]. We had a case who suffered from unilateral sudden deafness MRI showed Tumor on Occipital Lobe which is a Visual Central System, not an Auditory section perhaps because two Lobes are near to each other and because of correlation between them this occurred, so not only disorder in Central Auditory system may cause peripheral hearing loss malfunction in other parts of Brain may involve in causing hearing loss [3]. Neurofibromatosis type II is a genetic condition which may be inherited or may arise spontaneously. The main manifestation of the condition is the development of symmetric, benign brain tumors in the region of the cranial nerve VIII, which is the "auditory-vestibular nerve" that transmits sensory information from the inner ear to the brain and Auditory Brainstem Implant $(\mathrm{ABI})$ is a solution for individuals with hearing loss due to a nonfunctioning auditory nerve (Neurofibromatosis Type 2). Bypassing both the inner ear and the auditory nerve which stimulates the cochlear nucleus (CN) and provides users with a variety of hearing sensations to assist with sound awareness and communication [14]. There are some case studies which verified that non- tumoral cases such as cochlear hypoplasia, Mondini dysplasia who underwent Auditory Brainstem Implant could even understand speech with phone so if hearing without Cochlea and/or Auditory Nerve is possible it is obvious that hearing loss because of lesions in some part of brain without any lesion in Cochlea and/or Auditory Nerve is possible $[3,14]$.

We have seen a lot of patients with Sensory Neural hearing loss and normal Otoacoustic emissions this could be because of Auditory Neuropathy, lack of cooperation of patient during the Audiometry test, neural lesion, the collapse of the ear canal, but it is 
not always the case. There are some studies which showed normal or near normal OAE and compound action potential in those who suffer from the sensorineural hearing loss which indicated Cochlea and the Auditory nerve is intact therefore the reason of hearing loss could be because of lesions in the brain with or without mild lesion in Cochlea and/or Auditory nerve $[3,15]$ FMRI and PETSCAN show that Prefrontal and Temporal Lobes are more responsible for High-Frequency Auditory system and Cerebellum and brainstem typically subcortical areas are more in charge of understanding Low-Frequency Signals [5,8,13,16]. Reticular Formation and Prefrontal Lobe has a tremendous role in the hearing system typically in Speech recognition and selective hearing and the correlation between these part and other parts of the brain such as whole Cortex and Hippocampus are involved in speech recognition and Listening $[16,17]$.

\section{Backward auditory input theory}

It is impossible that our small Pinna and our tiny external ear canal is the only input to the auditory system, Cerebral Cortex and Brain could receive the sound from the Head [18]. It seems that there are two paths, Forward Auditory input signals which are the information from the external ear canal and the other one Backward Auditory input signals that is the information of signals which receive by Cerebral Cortex from the head [3]. Information from Forwarding Auditory input system reach to the Cochlear which is more Low frequencies and information from Backward Auditory input system comes down from the brain to approach to the Cochlear, typically more high-frequency information received by Backward Auditory input system $[3,19]$. The cochlea is the rendezvous between signals which come from Forwarding Auditory input, more low frequencies and signals which come from the Backward Auditory input, more high Frequencies and all information will be combined and organized and coding by Cochlea and will be sent to the Brain for final processing $[3,18,19]$. There are some examples which can confirm Backward Auditory input signals.

The First Maximum Conductive Hearing loss is 50 to 60 Decibels and not more than that and still we can hear [3]! Second studies showed that earmuff and ear plugs could not protect our ears from noise-induced hearing loss entirely [20]. Third, Spontaneous Otoacoustic Emissions (SOAE) s are sounds that are emitted from the ear with any external stimulation and are measurable with sensitive microphones in the external ear canal. If SOAEs is only because of Cochlea, why there are some studies which verified normal SOAE despite lesion in Cochlea? [21]. There is a possibility that SOAEs are because of backward Auditory input signals because there is a link between Tinnitus and SOAEs, nowadays we know that Tinnitus is more related to CNS, Limbic system and less related to Auditory system, so all these could confirm Backward Auditory input of sounds theory and the fact that our external ear canal is not the only input of sounds [22].

\section{Conclusion}

Cochlear Implant is a successful prosthesis not only because of Cochlear role in the hearing system because of transmission of sounds to the Brain and intensifies the Backward Auditory input system. Some of the contraindications of Cochlear Implant are Psychosis, Autism spectrum, patients who suffer from Central Auditory Processing Disorder (CAPD), so it means that hearing without CNS is impossible. Sensorineural hearing loss without lesions in Cochlea and/or Auditory Nerve is possible. Top-Down Auditory system disorders may cause disruption in Backward Auditory input system, receiving the sounds by Brain and cause hearing loss. For preventing Noise-Induced Hearing Loss new protections should design which can cover entire head and Auricles. There are a lot of similar sensorineural hearing losses which are the same in type and degree, but in inside the site of lesions may different.

\section{References}

1. MC Kenna MJ, Mills BG (1990) Ultrastructure and immune histochemical evidence of Measles virus in active Otosclerosis. Acta Otolaryngol Suppl 470: 130-139.

2. Aokim M, Wakaoka Y, Hayashi H, Nishihori T, Kuze B, et al. (2011) The relevance of hypothalamus- pituitary- aderno cortical axis- related hormones to the cochlear symptoms in Meniere's disease. Int J Audiol 50(12): 897-904

3. Bina A, Hourizadeh S (2015) The most important factors of causing Hearing Loss following central Auditory System Disorder and Central Nervous System (CNS) Disorder. J Otolaryngol ENT Res 2(6).

4. Alicia M Quesnel, Margaret Seton, Saumil N Merchant, Christopher Halpin, Michael J MC Kenna (2012) Third generation bisphosphonates for treatment sensorineural hearing loss in Otosclerosis. Otol Neurotol 33(8): 1308-1314.

5. L Clark Cox, Sandra L mc coy, Patricia A Tun, Arthur Wing field (2008) Monotonic Auditory Processing Disorder tests in the older adult. J Am Acad Audiol 19(4): 293-308.

6. Brody Rm, Nicholas BD, Wolf MJ, Marcin Kevich PB, Artz GJ (2013) Cortical deafness: a case report and review of the literature. Otol Neurotol 34(7): 1226-1229.

7. Walsh RM, Mu Punt JA, O Donoghue Gmrty GE (1994) Sudden contralateral deafness following Cerebellopontine angle Tumor surgery. Am J Otol 15(2): 244-246.

8. Syka J (2002) Plastic changes in the Central Auditory system After Hearing Loss. Restoration of Function and during learning. Physiol Rev 82(3): 601-636.

9. Mallur PS, Lal Wani AK (2007) Fluctuating corticosteroid- responsive auditory neuropathy/ dys synchrony is suggestive of Central nervous system pathology. Otol Neurotol 28(8): 1002-1004.

10. Celis Aguilar E, Macias Valle L, Coutinho De Toledo H (2012) Auditory Neuropathy secondary of Cryptococcal central nervous system infection in two immune compromised patients. Otolaryngol Head Neck Surg 147(3): 597-598.

11. Joseph P Pillion (2012) Speech Processing Disorder in Neural Hearing Loss. Case report in Medicine pp. 7.

12. Lee H, Whitman GT, Lim JG Lee SD, Park YC (2001) Bilateral sudden deafness as a prodrome of Anterior inferior cerebellar Artery Infarction Arch Neurol 58(8): 1287-1289.

13. Raksha A Mudar, Fatima T Husain (2016) Neural Alternations in Acquired Age- Related Hearing Loss. Front Pyschol 7: 828. 
14. Colletti V Auditory outcomes in tumor vs non -tumor patients fitted with Auditory Brainstem Implant. Adv Otorhinolaryngol 64: 167-85.

15. Ohashi T, Nishino H, Otsuka T, Koizuka I (2011) Recovery from adoption of the action potential in idiopathic Sudden Sensorineural Hearing Loss investigated using a paired- click Stimulation paradigm. Acta Otolaryngol 131(11): 1165-1171.

16. Yu Yf, Zhai F, Dai CF, Hu JJ (2011) The relationship between age- related hearing loss and synaptic changes in the hippocampus Exp Gerontol 46(9): 716-722.

17. Remy Pujol, Sam Irving (2016) Auditory messages are conveyed to the Brain via two types of pathway: The primary auditory pathway which exclusively carries messages from the Cochlea and the nonprimary pathway which is reticular pathway carries all types of sensory messages. Journey into the world of Hearing- Auditory Brain.
18. Bryan Gick, Donald d Derrick (2009) Aero- tactile integration in speech perception. Nature 462: 502-504.

19. Byung In Han, Howon Lee, Tae You Kim, Jun Seong Lim, Kyoung Sik Shin (2009) Tinnitus: Characteristics, mechanisms and Treatment J Clinic Neurol 5(1): 11-19.

20. Berger EH (1983) Laboratory attenuation of earmuffs and earplugs both singly and in combination. AM Ind Hyg Assoc J 44(5): 321-329.

21. W Clark, Do Kim, PM Zurek, BA Bohne (1984) Spontaneous Otoacoustic emissions in Chinchilla ear Canals: Correlation with histopathology and suppression by external tones. Hearing Research 16(3): 229-314.

22. Colletti V Auditory outcomes in tumor vs non -tumor patients fitted with Auditory Brainstem Implant. Adv Otorhinolaryngol 64: 167-185.

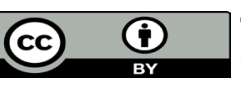

This work is licensed under Creative Commons Attribution 4.0 License

To Submit Your Article Click Here:

Submit Article

DOI: $10.32474 /$ SJO.2019.02.000133

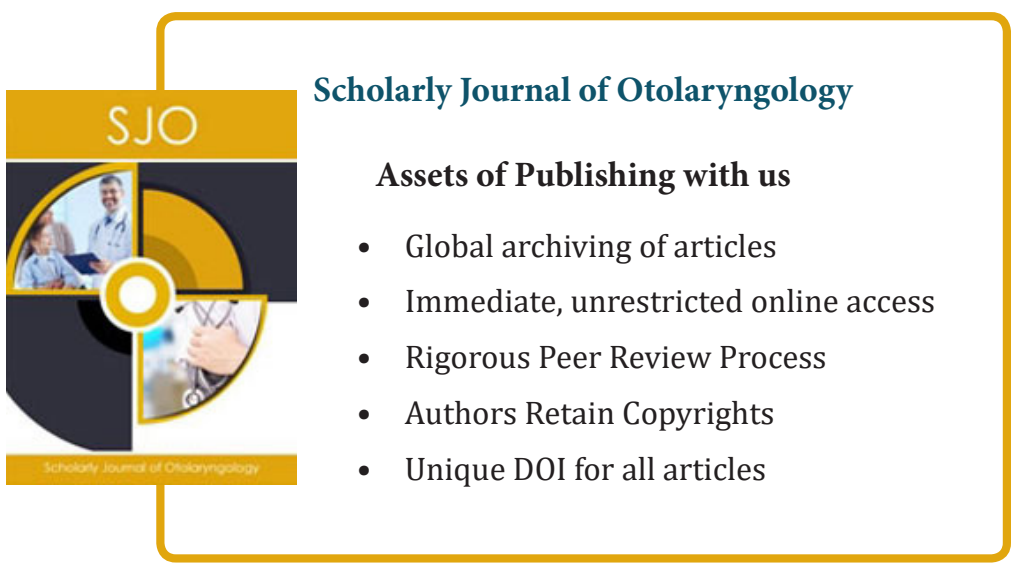

